Journal of Animal and Veterinary Advances 11 (9): 1455-1460, 2012

ISSN: $1680-5593$

(C) Medwell Journals, 2012

\title{
The MHC class I of Grass Carp (Ctenopharyngodon idellus) With Different Way to Present Peptide
}

\author{
Jia Zhen-Hu \\ College of Life Science, Shanxi Normal University, Linfen, 041004 Shanxi, P.R. China
}

\begin{abstract}
Major Histocompatibility Complex class I (MHC class I) proteins are critical for immune defenses against viruses. Polygeny and polymorphism of alleles contribute to the breadth of the immune response. In previous study, researchers showed that a MHC class I gene termed Ctid-AB190929 was found from a grass carp. It was a expressed gene from cDNA library. Now, characterization of a genomic clone from the same grass carp constructed fosmid genome library allows us to further analysis the $\mathrm{MHC}$ class I region. This clone carried whole $M H C$ class I and tapasin gene. This MHC class I termed Ctid-UBA0201 was the classical MHC class I by genomic analysis. But the amino acid sequences of Ctid-UBA0201 were differed from that of Ctid-AB190929. As lower vertebrates, appear to use a single expressed MHC class I locus to present a wide variety of peptide to antigen. The Ctid-UBA0201 could be the allelic gene of Ctid-AB190929. As the tapasin gene may be a unexpressed gene by genomic analysis, the Ctid-UBA0201 may present a wide variety of peptide without the tapasin gene to control the assembly. It may preserve genetic diversity which can be exchanged when the species face a new pathogen.
\end{abstract}

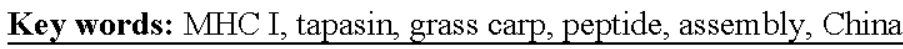

\section{INTRODUCTION}

Major Histocompatibility Complex class I (MHC class I) proteins play a fundamental role in the presentation of endogenously derived peptides to CTLs. MHC class $I$ genes are critical for immune defenses against viruses (Moon et al., 2005). Their genomic regions are the most dynamic part of the genome. Polygeny and polymorphism of their alleles contribute to the breadth of the immune response. The number of $\mathrm{MHC}$ class I loci can differ greatly in each species. Thus, far the genomic sequences of humans, mammals, reptiles, amphibians, birds and fish indicate that there are one or more class I loci in jawed vertebrates (Shiina et al., 1999; Flajnik and Kasahara, 2001; Kulski et al., 2002; Matsuo et al., 2002).

Teleost (bony) fish are the largest group of vertebrates, comprising about half of the total living vertebrates. As lower vertebrates, appear to use a single $\mathrm{MHC}$ class I locus to present a wide variety of peptide to antigen. Since, then the MHC class $I$ genes have been reported in at least nine species, at least in the trout and Atlantic salmon, it was shown definitively that there is only a single expressed MHC class I gene (Hansen et al., 1996; Shum et al., 2001).

Grass carp (Ctenopharyngodon idellus) as one of the most important freshwater fish species in China can service as a model fish representing not only the tradtional fish cultured in pond but also the fish cleaning the excessive grass in the river. In previous study, one expressed MHC class I gene named Ctid-AB190929 have been found in grass carp (Hao et al., 2006).

To further understand the MHC class I gene in grass carp, a genomic clone containing the $M H C$ class $I$ and tapasin gene was found out from a grass carp fosmid library. The MHC class I was termed Ctid-UBA0201.

\section{MATERIALS AND METHODS}

Screening of a fosmid genomic library for tapasin and MHC class I gene: The fosmid genomic library CIGF from a adult grass carp (fish-C) was previously constructed (Jia et al., 2010). The CIGF was screened using the primer pairs derived from a conserved region of $\mathrm{MHC}$ class I exon 4 common to cDNA sequences previously identified in the same individual fish. Screening of the library and identifing one clone containing tapasin and MHC class I gene clone GIGF-0405E6.

Sequencing strategy: Because preliminary sequencing suggested that tapasin and MHC class I gene could be in clone GIGF-0405E6. The clone was further sequenced by the Shotgun Method. First, fosmid DNA sample was extracted and broken up by ultrasonication. Subsequently, subcloned libraries of T-vectors were constructed from the clone GIGF-0405E6 as described 
previously by Taghian and Nickoloff (1996). The sequences of 1000 positive clones having an insert size of $2-3 \mathrm{~kb}$ from library were determined with the ABI PRISM Big Dye Primer Cycle Sequencing Ready Reaction kit (Applied Biosystems) and the ABI 377 DNA sequencer using $\mathrm{T} 7$ and SP6 sequence primers (The Beijing Genomics Institute).

Finally, determining the order of the subcloning fragment in the final $33 \mathrm{~kb}$ contig was accomplished with Phrap (www.phrap.org/consed/consed.html \#howToGe). The sequence of the entire fosmid clone is deposited in GenBank under the accession number EF584535.

Sequence analysis: The edited GIGF-0405E6 final sequence were analyzed for coding regions using GENSCAN (http://genes.mit.edu/GENSCAN.html) and through homology searches using BLAST. MHC class I gene within the clones were compared with cDNA previously published. Polymorphisms and the phylogenetic tree were analyzed using DDBJ .

Promoter analysis: The promoter was analyzed using Genomatix MatInspector Software. Grass carp sequences were also aligned to known $\mathrm{MHC}$ class I Promoter zebrafish using ClusalW and conserved sites within the promoter regions were identified manually.

\section{RESULTS AND DISCUSSION}

The clone GIGF-0405E6 was further sequenced by the Shotgun Method. As an insert size of $2-3 \mathrm{~kb}$ was needed to construct subcloned libraries of T-vectors. The DNA sample of GIGF-0405E6 was extracted and broken up by ultrasonication. As Fig. 1 shows the size of DNA sample has been $2-3 \mathrm{~kb}$ by ultrasonication three times and $1-2 \mathrm{~kb}$ by ultrasonication five times.

Selecting the insert size from $T$-vectors for sequencing: Because subcloned libraries of T-vectors were constructed from the clone GIGF-0405E6 and the insert size was $2-3 \mathrm{~kb}$ by ultrasonication. Researchers selected insert size of $2-3 \mathrm{~kb}$ to sequence. As Fig. 2 shows the number of $1,3,4,7,8,9$ and 10 could be select to sequence.

The homology analysis with zebrafish: The edited GIGF-0405E6 final sequence were analyzed for coding regions through homology searches using BLAST (Fig. 3). The GIGF-0405E6 carried whole MHC class I and tapasingene.

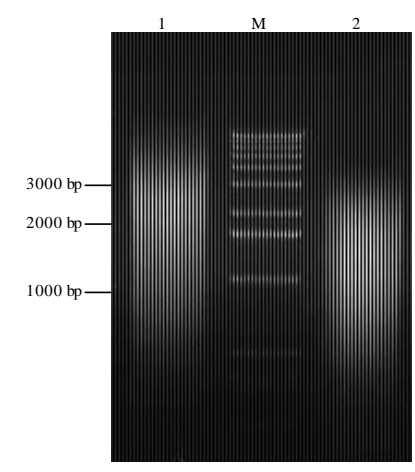

Fig. 1: Ultrasonication of the clone GIGF-0405E6; M: $1 \mathrm{~kb}$ marker; 1 : Three times by ultrasonication and 2 : Five times by ultrasonication

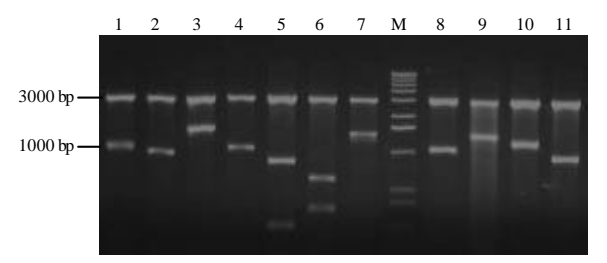

Fig. 2: Enzymatic digestion of the recombination Tvectors by restricted enzymic EcoRI; M: $1 \mathrm{~kb}$ marker and 1-11: Recombination T-vectors of digested by EcoRI

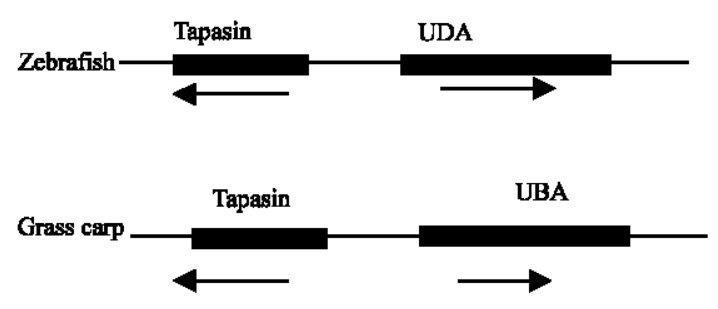

Fig. 3: Predicted genes of GCFL-0405E6 with zebrafish. Arrows indicate the transcriptional orientation of the gene

Organization of the exon-intron of Ctid-UBA gene: A complete contig of $33 \mathrm{~kb}$ contained part of the tapasin and Ctid-UBA0201 genes in EF584535 (www.ncbi.nlm.nih. gov/nuccore/EF584535.1) (Fig. 4). The tapasin gene started at $7160 \mathrm{bp}$ and ended at $1382 \mathrm{bp}$, consisting of 5778 bp. Exon 6 contained 93 bp. Exon 1 was 61 bp and encoded a signal peptide. Exon 2 was 196 bp. Intron 1 was 3644 bp. Exon 3 contained 249 bp. Intron 2 was 88 bp. Exon 4 was 360 bp.Intron 3 was 165 bp. Exon 4 was 360 bp. Intron 4 was 153 bp. Exon 5 was 93 bp. Intron 5 was 93 bp. Exon 6 was 4056 bp. Intron 6 was 249 bp.

The Ctid-UBA0201 gene started at $4505 \mathrm{bp}$ and ended at 20,980 bp, consisting of 16,475 bp. Exon 1 contained $49 \mathrm{bp}$ and encoded a signal peptide. Exon 2 was 
$261 \mathrm{bp}$ and encoded the $\alpha 1$ domain. Intron 1 was $1018 \mathrm{bp}$. Exon 3 contained 276 bp and encoded the $\alpha 2$ domain. Intron 2 was 12,285 bp. Exon 4 was 288 bp and encoded the $\alpha 3$ domain. Intron 3 was 1572 bp. Exon 4 was 99 bp and encoded the TM domain. Intron 4 was 335 bp. Exon 5 was 18 bp and encoded the CY1 domain. Intron 5 was 172 bp. Exon 6 was 53 bp and encoded the CY 2 domain. Intron 6 , located between exon 6 and 7 was $249 \mathrm{bp}$. The Ctid-UBA 0201 gene encoded 342 aa (Fig. 5).

The promoter in Ctid-UBA 0201: Promoter organization was analyzed in the genomic sequences $800 \mathrm{bp}$ before the start codons of the Ctid-UBA0201 gene. GAAA motifs were found at positions $-733,-654,-384$ and -314 . An IFNStimulated Regulatory Element (ISRE) sequence was

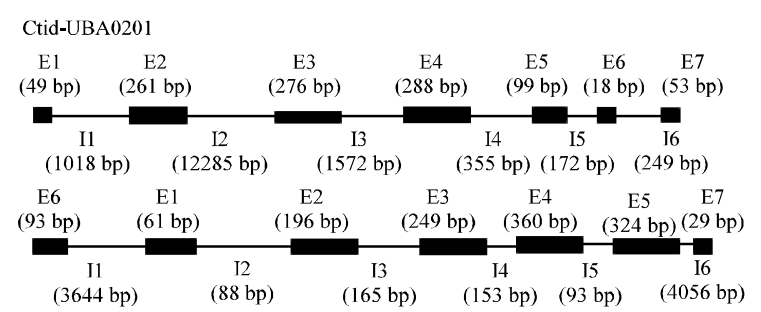

Fig. 4: The exon-intron organization of Ctid-UBA0201and tapasin. The exon sizes are noted above the sequences and the intron sizes are noted

\begin{tabular}{|c|c|c|c|c|c|}
\hline-780 & -770 & -760 & -750 & -740 & -730 \\
\hline TCACAGTGCA & TTAACAAATA & CAACTTTTGA & TTTAATAATG & TATCAGTAAA & TGTTEAAAATT \\
\hline-720 & -710 & -700 & -690 & -680 & -670 \\
\hline AACATTAAGA & TTAATAAATG & CTGTTGAAGT & ATTGTTCATT & CTTAGTACAT & GTAGTTAACA \\
\hline-660 & -650 & -640 & -630 & -620 & -610 \\
\hline CATGATAACA & AATGAAAGCT & TATTGTAAAG & TGTTACCAAT & ATCACTATAT & AACAAACAAG \\
\hline-600 & -590 & -580 & -570 & -560 & -550 \\
\hline GTAAATTAAT & ACAATGCATT & GTATCAACAC & TATTGAAGTG & AGCTGTAACT & TGTAACGCTA \\
\hline-540 & -530 & -520 & -510 & -500 & -490 \\
\hline TTTAAAAAAG & ATGGACATCG & GTTTATAATT & TGTACATGCA & TTTATTTTGT & TAATCAAATT \\
\hline-480 & -470 & -460 & -450 & -440 & -430 \\
\hline AATTGTTCAA & AATAATTTTT & ATAATAACTA & CCAACTCCCA & TAAACAAACA & TGATATTTCT \\
\hline-420 & -410 & -400 & -390 & -380 & -370 \\
\hline ATTATGTGAA & CATTTTAAGT & AAATGTTATA & TATTAAATTA & AAAGAAAATC & TGTTGATAAA \\
\hline-360 & -350 & -340 & -330 & -320 & -310 \\
\hline GATGACACCA & GCATGCTTTA & ACCTGGTCAC & AAATCAGATA & ATAACCCTGA & GGAGAAATGT \\
\hline-300 & -290 & -280 & -270 & -260 & -250 \\
\hline TGACTTTTCT & AACTTTATTT & ATTTATTTAT & TTATTTATTT & ATTTACCTTT & AATATGTCCT \\
\hline ISRE & -230 & -220 & -210 & S-box & -190 \\
\hline TTATATTTGT & GCACAAGACT & GCTGCCGCCA & CTATCCTGAT & CAACCCAGTT & AAGGAAGTAG \\
\hline-180 & -170 & X2-box & l-box & EnhB & -130 \\
\hline CTCTTAGTAA & CTTGTGACGT & TGAACGTACC & TGGAATATCT & ATTGGCTGGA & ATTTCAGAGA \\
\hline-120 & -110 & -100 & -90 & -80 & -70 \\
\hline CTATTTAGGC & GTTCTCTCA $\underline{G}$ & $\underline{\boldsymbol{A A A} \text { CATTTCA }}$ & TCAGTATATC & GCCAGACATC & TGAAACGTCT \\
\hline-60 & -50 & -40 & -30 & TATA & -10 \\
\hline AGAACAAATA & $\begin{array}{l}\text { TTTCAATTAA } \\
1\end{array}$ & TTTCTCTAGA & ACGATTTTTA & CAAAAACTGT & TTCTCATGCA \\
\hline ACAGCAAGGA & TG & & & & \\
\hline
\end{tabular}

found at position -242. The SXY box, composed of an S-box, X1 X2 and $\operatorname{EnhB}(\mathrm{Y})$ is a common promoter for expression of the MHC class $I, M H C$ class $I I$ and $\alpha 2 m$ genes and was found at positions $-203,-164$ and -146 in Ctid-UBA 0201. The results indicate that a common promoter region exists in Ctid-UBA0201 gene.

High polymorphism of the MHC class I of grass carp: To investigate the allelic polymorphism of Ctid-UBA 0201 genes, 26 grass carp were used to clone the MHC class I genes using allele-specific primers vertebrates. The $\alpha 1$ and $\alpha 2$ domains of the expressed MHC class $I$ genes contained 85-89 and 91-93 aa, respectively. The amino acid identities of $\mathrm{MHC}$ class I ranged from 64.53-83.63\%. The motif ( $Y, Y, R, T, K, W, Y$ and $Y$ ) which is crucial for peptide binding was showed with asterisks. Dashes indicate sequence identity (Fig. 6).

In previous study, researchers have constructed and characterized the genome fosmid library with excellent quality of grass carp and named this fosmid clone library as CIGF. From this library, clone GIGF-0405E6 had been screened. Though the sequence of the entire clone GIGF0405E6 could be get by clone walking, it needs too much time (Bradeen et al., 2003). Subcloned library of T-vectors was constructed from the clone GIGF-0405E6. About 1000 positive clones having an insert size of $2-3 \mathrm{~kb}$ from this

Fig. 5: The promoter in Ctid-UBA 0201. Putative transcription factorbinding sites are indicated. Enhancer elements including the ISRE are underlined and conserved motifs involved in the constitutive expression of MHC class I sequences are indicated above in bold 


\begin{tabular}{|c|c|c|c|}
\hline a1 domain & $*$ & \multicolumn{2}{|r|}{ Identity $(\%)$} \\
\hline \multirow[t]{2}{*}{ Ctid-UBA0201 } & $\begin{array}{c}\text { GTHSLKYLYTAVSGDIDFPEFTAVGLVDDEQFMYFDSNIKKAVPKTEWIRQN } \\
*\end{array}$ & & \\
\hline & VGADYWDSETQSDIVSHRAFKMNIQIAMERFNQSKG & 88 & \\
\hline CtidUBA190929 & 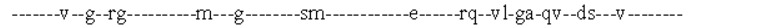 & 87 & 76.14 \\
\hline Ctid-AB190930 & ---tyq-f---a--lpn--k-v---m-ng--in-y--stme--v-ad-vkgi-npte-kn... iwlg-qqtyiegvk-v-n-y-h-dv & 85 & 37.50 \\
\hline Ctid-UAA101 & - & 87 & 80.68 \\
\hline Ctid-UAA102 & -------V --g--rg----------m----g--i-----sm---------------c--r---nh-ghsq--------------- & 87 & 79.55 \\
\hline Ctid-UAA103 & 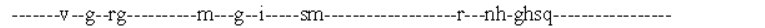 & 87 & 80.68 \\
\hline Ctid-UAA104 & ------v --g--rg---------m---g--1-----sm--------------r---nh-ghsq------------- & 87 & 80.68 \\
\hline Ctid-UAAA105 & 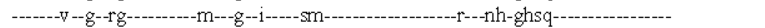 & 87 & 80.68 \\
\hline Ctid-UBA101 & 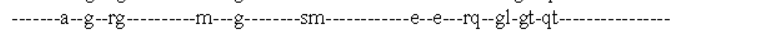 & 87 & 78.41 \\
\hline Ctid-UCA101 & -----r-f--..--------------g--------m--------m---------iqn-nl-ga-qv--d----k------- & 85 & 77.27 \\
\hline Ctid-UCA102 & ----r-f--.. & 85 & 77.27 \\
\hline Ctid-UCA103 & --or-f-1--1 & 87 & 80.68 \\
\hline Ctid-UDA.101 & -k-----f-----n-- & 87 & 86.36 \\
\hline Ctid-UDA102 & 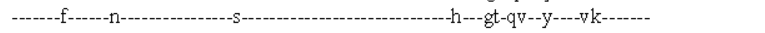 & 87 & 86.36 \\
\hline Ctid-UEA101 & ---tyq-f---a--lpn--k-v---m-ng--in-y--stme--v-ad-vkgi-npte-kn...iwlg-qqtyiegvk-v-n-y-h-dv & 85 & 37.50 \\
\hline Ctid-UEA102 & --tyq-f---a--lpn--k-v---m-ng--in-y--stme--v-ad-vkgi-npte-kn... iwlg-qqtyiegvk-v-n-y-h-dv & 85 & 37.50 \\
\hline Ctid-UFA101 & -m-----ff-g-------y------eg--y-------q---------e-----r---nmv gy-qn---s---1k-----a & 87 & 73.86 \\
\hline Ctid-UFA102 & -m-----ff-g--------y------eg --y-------q----------e-----r---nmv gy-qn---s---1k-----a & 87 & 37.50 \\
\hline Ctid-UGA101 & 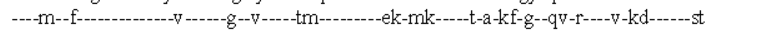 & 89 & 73.03 \\
\hline Ctid-UHA101 & 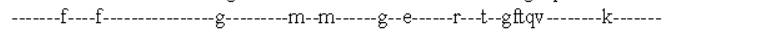 & 87 & 81.82 \\
\hline Ctid-UHA102 & 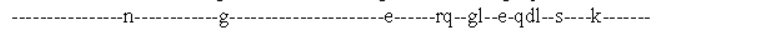 & 87 & 84.09 \\
\hline Ctid-UIA101 & -------V --g--rg---------m---g--i-----sm--------k-------r---nh-ghsq------------- & 87 & 79.55 \\
\hline Ctid-UIA102 & 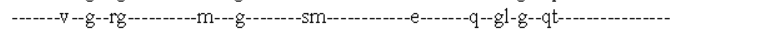 & 87 & 81.82 \\
\hline Ctid-UJA101 & 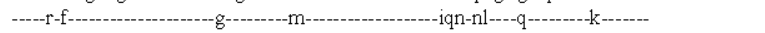 & 87 & 86.36 \\
\hline Ctid-UJA102 & - & 87 & 88.64 \\
\hline Ctid-UJA103 & 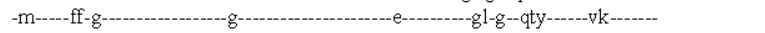 & 87 & 82.95 \\
\hline Ctid-UKA101 & 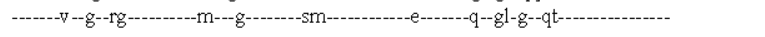 & 87 & 81.82 \\
\hline \multicolumn{4}{|l|}{$\alpha 2$ domain } \\
\hline \multirow[t]{2}{*}{ Ctid-UBA0201 } & VHTFQEMYGCEWDDQT GTTDGFRQEGYDGEDFLSLDLKEERWFSPVC & 93 & \\
\hline & TVHKWNNDRADLEGRKHYFSTECIEWLKKYLEYGKSSLQKTV & & \\
\hline Ctid-UBA190929 & g---w-n-----ln-d---q--y-ya------v---kntlt-taanp-a-i-k---ea.1-va-qn-g-lent-------va---dt-erk & 91 & 51.06 \\
\hline Ctid-AB190930 & g---w------ln-d---q-yg-y----a--v --kntlt-taanp-a-i-k---ean--ia-qy-v-len--------vg---dt-erk & 92 & 53.19 \\
\hline Ctid-UAA101 & ----w-n-----ln- d---q--y-ya------v---kntlt-taanp-a-i-k---ea l-va-qn-g-lent-------va---dt-erk & 91 & 51.61 \\
\hline Ctid-UAA102 & g---w-n-----ln-d---q--y-ya------v ---kntlt-taanp-a-i-k---ea l-va-qn-g-lenty------va---dt-erk & 91 & 50.00 \\
\hline Ctid-UAA103 & g---w-n-----ln-d---q--y-ya-----v---kntlt-taanp-a-i-k---ea. 1-va-qn-g-lent-------va---dt-erk & 91 & 51.06 \\
\hline Ctid-UAA104 & g---w-n-----ln-d---q--y-ya------v---kntlt-taanp-a-i-k---ea 1-v a-qn-g-lent-------va a---dt-erk & 91 & 51.06 \\
\hline Ctid-UAA105 & g---w-nv----ln-d---q--y-ya------v--kntlt-taanp-a-i-k---ea. l-va-qn-g-lent-------va---dt-erk & 91 & 50.00 \\
\hline Ctid-UBA101 & g----k----ln-d---q--y-y----a--v --kntlt-taanp-a-i-k---eat-faate-v-lent-v-----va---dt-erk & 91 & 50.00 \\
\hline Ctid-UCA101 & g--sl-w-----1--d --kr-ym-y-------a--kstlt-taa-p-ati-kk-1dstg-eansdnn-ldni-----n--v d---dt-mrk & 92 & 44.68 \\
\hline Ctid-UCA102 & g--s--w-----1-- d--kr-ym-y-------a-kstlt-taa-p-ati-kk-1dstg-eansdnn-ldnt-----n--vd---dt-mrk & 92 & 45.74 \\
\hline Ctid-UCA103 & g--s1-w----1-- d--kr-ym-y-------a--kstlt-taa-p-ati-kk-1dstg-eansdnn-1dnt-----n--vd---dt-mrk & 92 & 44.68 \\
\hline Ctid-UDA101 & g--1--w-----1--.d-skr-ym-y----d---t-kstrs-taanp-ati-kg--dstgtka-vdsn-lent-----n--v d---dt-trk & 92 & 43.62 \\
\hline Ctid-UDA102 & g-1--w-w---1--.d-skr-ym-y----d---t-kstrs-taanp-ati-kg--dstgtka-ydsn-lent-----n--v d---dt-trk & 92 & 43.62 \\
\hline Ctid-UEA101 & g---w-1-----ln.. d---q-yf-f-------v---kntvt-taanp-a-i-k---ean--ia-qq-e-lent----v---vg---dt-erk & 91 & 51.06 \\
\hline Ctid-UEA102 & g---w-1----ln. d---q-yf-f------v---kntvt-taanp-a-i-k---ean--ia-qq-e-lent----v---vg---dt-erk & 91 & 51.06 \\
\hline Ctid-UFA101 & g---w-v -----ln-d----qeyy-f----a--v---kstlt-taaspeaai-kn--ea---yta---e-lenr----vq--v g---dt-erk & 92 & 46.81 \\
\hline Ctid-UFA102 & g---w-v -----ln-d----q-yy-f----a--v ---kstlt-taasp eaai-kn--ea---yta---e-lenr----vq--vg---dt-erk & 92 & 47.87 \\
\hline Ctid-UGA101 & g---y-1-----n---qakgai--y-f----yv i--w--q-yi-s----11saqr--e e-n--a--n-1-ni-----q-wq----i-e-q & 92 & 56.38 \\
\hline Ctid-UHA101 & 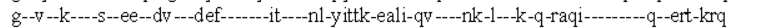 & 93 & 54.26 \\
\hline Ctid-UHA102 & g--v --q----s--ee--dv ---dkf-------it----nl-yittk-ealt-qv----nk-1--kk-q-raqi-------qq-ekt-kre & 93 & 54.26 \\
\hline Ctid-UIA101 & g---v-n------e--aasa-d-y-------a-h--1--v------fl-tq---dnk-e--ly-q-y-nq-t------q-----e-- & 93 & 65.96 \\
\hline Ctid-UIA102 & g---v-n------e--aasa-d-y-------a-h---1--v-----fl-tq---dnk-e--ly-q-y-nq-t------q-----e-- & 93 & 65.96 \\
\hline Ctid-UJA101 & g-o- & 93 & 82.98 \\
\hline Ctid-UJA102 & 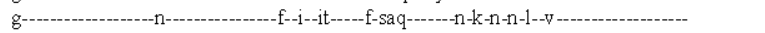 & 93 & 65.96 \\
\hline Ctid-UJA103 & 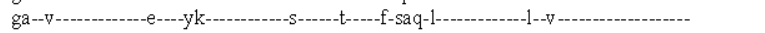 & 93 & 82.98 \\
\hline Ctid-UKA101 & g---w-v -----ln-.d---q-yy-f----a--v ---kstlt-taaspeaai-kn--ea---yta---e-lenr----vq--vg---dt-erk & 92 & 47.87 \\
\hline
\end{tabular}

Fig. 6: Comparison of the PBD domains of Ctid-UBA0201 amino acid sequences to the MHC class I of other grass carp

library were selected to sequence. It ensured the each bases of clone GIGF-0405E6 was covered 8-10 times. So, the sequence of the entire fosmid clone had excellent quality.

Similar to chickens, ducks and mammals, the MHC class I regions in most bony fish are composed of seven exons and six introns (Hansen et al., 1999). In this study, the Ctid-UBA0201 gene also has seven exons and six introns. Some reports have confirmed that in bony fish the MHC class $I$ genes can also confer resistance to pathogens of the bacterial and viral (Chen et al., 2010).
The polymorphic residues of the Peptide-Bindingdomain (PBD) of MHC class I are the key to resist the pathogens. The PBD is made up of the $\alpha 1$ and $\alpha 2$ domains. Comparison of the PBD domains of Ctid-UBA0201 amino acid sequences to the classical $\mathrm{MHC}$ class I of other grass carp reported in the previous study (Yang et al., 2006). The $\alpha 1$ and $\alpha 2$ domains of the Ctid-UBA0201 genes contained 85-89 and $91-93$ aa, respectively. The amino acididentities of Ctid-UBA0201 ranged from $64.53-83.63 \%$. The motif ( $Y, Y, R, T, K, W, Y$ and $Y$ ) which is crucial for peptide binding was conserved in the Ctid-UBA0201 
genes. These finding imply that Ctid-UBA 0201 gene has the characteristics of the classical MHC class I gene.

The SXY is generally believed to be the shared promoter for MHC class I, MHC class II and $\alpha 2 \mathrm{~m}$ (Gobin et al., 1998). Analysis of the promoter region of Ctid-UBA 0201 showed that the promoter had this original feature in bony fish and then evolved in ducks, mammals and humans. The transcriptional regulatory regions were composed of five conserved domains: S-box, X1 3 2, EnhB (Y), GAAA and ISRE motifs. This finding implies that Ctid-UBA 0201 gene can express. Because the CtidAB190929 and Ctid-UBA0201 was came from same grass carp and the Ctid-AB190929 was a expressed $M H C$ class $I$ gene from cDNA library. The amino acid sequences of Ctid-UBA0201 were differed from that of Ctid-AB190929. As lower vertebrates, appear to use a single MHC class I locus to present a wide variety of peptide to antigen. The Ctid-UBA0201 could be the allelic gene of Ctid-AB190929.

In all species studied to date, the MHC is a large chromosomal region, containing many genes encoding proteins of immunological importance. Several cofactors are essential for proper MHC class I molecules assembly, such as proteasome, calnexin, calreticulin, ERP57, tapasin and TAP (Grandea and Van Kaer, 2001). Tapasin is supposed to play an important role in the quality control of $\mathrm{MHC}$ class I molecules assembly in endoplasmic reticulum. It is still unclear how tapasin controls the assembly of $\mathrm{MHC}$ class I molecules in ER. But pech argues that tapasin-mediated bridging of TAP-class I complexes is not necessarily sufficient for peptide loading (Peh et al., 2000).

In this study, researchers agree with the view of pech. The Ctid-UBA 0201 has the characteristics of the classical MHC class I gene but the tapasin gene may be a unexpressed gene by genomic analysis. So, the CtidUBA0201 may present a wide variety of peptide without the tapasin gene to control the assembly. It may preserve genetic diversity which can be exchanged when the species face a new pathogen.

\section{CONCLUSION}

The Ctid-UBA 0201 gene is the classical $M H C$ class I gene and the allelic gene of Ctid-AB190929. It may present a wide variety of peptide without the tapasin gene to control the assembly.

\section{ACKNOWLEDGEMENT}

The study was supported by a grant from the Natural Science Foundation of China (Grant No: 30371098).

\section{REFERENCES}

Bradeen, J.M., S.K. Naess, J. Song, G.T. Haberlach and S.M. Wielgus et al., 2003. Concomitant reiterastive $\mathrm{BAC}$ walking and fine genetic mapping enable physical map development for the broad-spectrum late blight resistance region, RB. Mol. Genet. Genomics, 269: 603-611.

Chen, W., Z. Jia, T. Zhang, N. Zhang and C. Lin et al., 2010. MHC class I presentation and regulation by IFN in bony fish determined by molecular analysis of the class I locus in grass carp. J. Immunol., 185: 2209-2221.

Flajnik, M.F. and M. Kasahara, 2001. Comparative genomics of the MHC: Glimpses into the evolution of the adaptive immune system. Immunity, 15: 351-362.

Gobin, S.J., A. Peijnenburg, M. van Eggermond, M. van Zutphen, R. van den Berg and P.J. van den Elsen, 1998. The RFX complex is crucial for the constitutive and CIITA-mediated transactivation of $\mathrm{MHC}$ class I and beta2-microglobulin genes. Immunity, 9: 531-541.

Grandea, A.G., and L. Van Kaer, 2001. Tapasin: An ER chaperone that controls MHC class I assembly with peptide. Trends Immunol, 22: 194-199.

Hansen, J.D., P. Strassburger and L. Du Pasquier, 1996. Conservation of an alpha 2 domain within the teleostean world, $\mathrm{MHC}$ class I from the rainbow trout Oncorhynchus mykiss. Dev. Comp. Immunol., 20: $417-425$.

Hansen, J.D., P. Strassburger, G.H. Thorgaard, W.P. Young and L. Du Pasquier, 1999. Expression, linkage and polymorphism of MHC-related genes in rainbow trout, Oncorhynchus mykiss. J. Immunol., 163: 774-786.

Hao, H.F., T.Y. Yang, R.Q. Yan, F.S. Gao and C. Xia, 2006. cDNA cloning and genomic structure of grass carp (Ctenophayngodon idellus) beta2-microglobulin gene. Fish Shellfish Immunol, 20: 118-123.

Jia, Z.H., C.Y. Lin, T.Y. Yang, Y.N. Jiang and C. Xia, 2010. Construction and characterization of grass carp (ctenopharyngodon idellus) fosmid library. Agric. Sci. China, 9: 1347-1352.

Kulski, J.K., T. Shiina, T. Anzai, S. Kohara and H. Inoko, 2002. Comparative genomic analysis of the MHC: The evolution of class I duplication blocks, diversity and complexity from shark to man. Immunol. Rev., 190: 95-122.

Matsuo, M.Y., S. Asakawa, N. Shimizu, H. Kimura and M. Nonaka, 2002. Nucleotide sequence of the MHC class I genomic region of a teleost, the medaka (Oryzias latipes). Immunogenetics, 53: 930-940. 
Moon, D.A., S.M. Veniamin, J.A. Parks-Dely and K.E. Magor, 2005. The MHC of the duck (Anas platyrhynchos) contains five differentially expressed class I genes. J. Immunol., 175: 6702-6712.

Peh, C.A., N. Laham, S.R. Burrows, Y. Zhu and J. McCluskey, 2000. Distinct functions of tapasin revealed by polymorphism in MHC class I peptide loading. J. Immunol., 164: 292-299.

Shiina, T., G. Tamiya, A. Oka, N. Takishima and H. Inoko, 1999. Genome sequencing analysis of the $1.8 \mathrm{Mb}$ entire human MHC class I region. Immunol. Rev., 167: 193-199.
Shum, B.P., L. Guethlein, L.R. Flodin, M.A. Adkison and R.P. Hedrick et al., 2001. Modes of salmonid MHC class I and II evolution differ from the primate paradigm. J. Immunol., 166: 3297-3308.

Taghian, D.G. and J.A. Nickoloff, 1996. Subcloning strategies and protocols. Methods Mol. Biol., 58: $221-235$.

Yang, T.Y., Hao H.F., Jia Z.H., Chen W.H. and C. Xia, 2006. Characterisation of grass carp (Ctenopharyngodon idellus) MHC class I domain lineages. Fish Shellfish Immunol., 21: 583-591. 